\title{
Jenseits des Abstinenzparadigmas - Ziele in der Suchttherapie
}

\author{
Daniel Meili \\ Susan Dober \\ Edgar Eyal
}

Beyond Abstinence Paradigm - Goals in Drug Therapy

\section{Zusammenfassung}

Nach medizinischen Kriterien muss Abhängigkeit von „harten“ Drogen als chronische Krankheit verstanden werden, welche oft einen tödlichen Ausgang hat. Therapeutische Maßnahmen dürfen daher nicht primär an der Vorstellung der Heilung gemessen werden, die oft mit Abstinenz gleichgesetzt wird. Das Realitätsprinzip verlangt umsetzbare Ziele.

Das basale Ziel der Behandlungen ist, das Überleben zu ermöglichen. Um das übergeordnete Ziel der Verbesserung der Lebensqualität zu erreichen, bedarf es darauf ausgerichteter gemeinsam erarbeiteter realistischer Schritte. Die Modifikation und die Eigenkontrolle des Drogenkonsums sind meist ein hilfreicher Bestandteil dieses Prozesses.

Neben dem Begriff der substitutionsgestützten Behandlung wird der Terminus der abstinenzgestützten Behandlung eingeführt, der mithelfen kann, die Suchtmedizin aus der Sackgasse des Abstinenzparadigmas hinauszuführen. Diese beiden Behandlungsmethoden sollten sowohl ambulant als auch stationär angeboten werden.

\section{Schliuisselwörter}

Substitution · Abstinenz · Drogenkonsum · Opiatabhängigkeit · Therapieziele

\section{Abstract}

According to medical criteria, addiction to "hard" drugs must be understood as a chronic and often terminal disease.

Therapeutic approaches should not be primarily measured by the concept of a cure, which is often equated with abstinence. Reality requires realistic goals.

The main goal is to enable the addicts to survive. Achieving the higher goal of improving quality of life requires realistic steps which keep this in mind and are developed together with the addict. The modification of and self-control in drug use is usually a helpful part of this process.

In addition to the term substitution-assisted treatment, the term abstinence-assisted treatment will be introduced. This concept could help lift drug therapy out of the dead end of the abstinence paradigm. Both methods should be offered in an inpatient as well as an outpatient setting.

\section{Key words}

Substitution - abstinence $\cdot$ drug use - opiate dependence $\cdot$ therapeutic goals

\section{Einleitung}

Die folgenden Ausführungen beziehen sich auf das Feld der illegalen Drogen mit der „harten“ Droge Heroin als Leitsubstanz. Die Überlegungen schließen aber auch Kokain mit ein und Analogie- schlüsse zu weiteren illegalen und legalen Drogen lassen sich ziehen.

Auch nach über 150-jähriger Auseinandersetzung der Medizin mit der Suchtthematik - später der Psychologie, der Sozialarbeit,

Institutsangaben

ARUD Zürich, Arbeitsgemeinschaft für risikoarmen Umgang mit Drogen

Korrespondenzadresse

Dr. med. Daniel Meili · ARUD Zürich · Konradstr. 1 · 8005 Zürich · Schweiz · E-mail: d.meili@arud-zh.ch

Bibliografie

Suchttherapie 2004; 5: 2-9 @ Georg Thieme Verlag Stuttgart • New York ·

ISSN 1439-9903 · DOI 10.1055/s-2004-812915 
der Sozialpädagogik und anderen - wird bis heute mehrheitlich von der Vorstellung ausgegangen, die Suchttherapie habe den Abhängigen und die Gesellschaft von der Sucht zu befreien. Dieser Leitsatz hat seine Gültigkeit bewahrt, auch wenn er oft etwas modifiziert und in ein humaneres Mäntelchen gehüllt daherkommt. Bei Opiatabhängigkeit wird die Substitution als Mittel zur Schadensverminderung weit gehend akzeptiert. Das Kredo heißt dann: Wenn schon nicht abstinent, so doch abstinent von Beikonsum - und langfristig lauert die Abstinenz weiterhin als unumgängliches Ziel. Man könnte diese Haltung „Abstinenzparadigma light" nennen. Sei es ambulant oder stationär, das Abstinenzparadigma in der Suchttherapie lebt weiter.

Ein wichtiger Grund für das Festhalten an der Abstinenz als oberstes Behandlungsziel dürfte sein, dass wohl in keinem anderen Gebiet der Medizin derart viele verschiedene, auch konträr positionierte Kräfte bei der Gestaltung des Hilfsangebots mitmischen wie im Bereich der illegalen Drogen. Weit gehend moralische und normative Ansätze, die Strömungen aus der Bevölkerung, der politischen Parteien, der Sozialämter, der Justiz und der Polizei entstammen, bestimmen die Rahmenbedingungen von Behandlungen. Dies wird dann Drogenpolitik genannt. Kein Internist würde sich in seinem Gebiet Ähnliches gefallen lassen, kein Psychiater würde Derartiges in der Behandlung schizophrener Menschen akzeptieren. Es gibt keine Diabetespolitik und keine Schizophreniepolitik.

Sucht (oder Abhängigkeit) ist per definitionem eine chronische Erkrankung [1, 2]. Um vom Abstinenzparadigma wegzukommen, kann man sich bei der Behandlung von drogenabhängigen Menschen lediglich an zwei Grundzielen in der Behandlung chronischer Erkrankungen orientieren: erstens der Reduktion der wichtigsten Schädigungen und zweitens der Verbesserung der Lebensqualität. Generell müssen Zielsetzungen realistischerweise erreichbar sein (Realitätsprinzip). Deren Festlegung und die Planung zur Umsetzung müssen gemeinschaftlich zwischen den zu Behandelnden und den Therapierenden festgelegt und akzeptiert werden (Gemeinschaftsprinzip), um stabile Veränderungen zu erlangen. Wie in der Folge gezeigt wird, kommt es in der Behandlung von Opiatabhängigen zu einer grundlegend anderen Gewichtung des Angebots, wenn die Reduktion der Mortalitätsrate und die Förderung der Lebensqualität ins Zentrum des therapeutischen Schaffens rücken.

\section{Zielsetzungen in der Suchttherapie anhand von Prognose und Beeinflussbarkeit}

Bei Diskussionen unter Fachkräften muss immer wieder erstaunt festgestellt werden, wie Zielsetzungen mit derselben Patientengruppe um 180 Grad divergieren können. Das Spektrum reicht von der Abstinenz als unabdingbares oberstes Ziel bis zur Forderung der kompletten Freigabe der Drogen, da der Drogenkonsum in der Verantwortung des Einzelnen liegen müsse. Wie soll es da möglich sein, den Abhängigen Ziele vorzugeben oder erst noch gemeinsam mit ihnen zu erarbeiten?

Welche Ziele haben überhaupt eine Chance, erreicht zu werden? Schließlich sind Ziele nur dann sinnvoll, wenn auf den Prozess ein Einfluss ausgeübt werden kann, der eine Veränderung in Richtung der Zielumsetzung bewirkt (Realitätsprinzip).

Im psychosozialen Bereich (Wohnen, Arbeit, Kriminalität) ist die positive Einflussnahme durch therapeutische Maßnahmen bei Drogenabhängigen, seien sie stationär oder ambulant, weit gehend unbestritten. Wenden wir uns also auf der einen Seite der unbekannteren Mortalitätsrate zu, auf der anderen Seite dem Drogenkonsum bzw. der Abstinenz. Die Ausführungen dazu beziehen sich zu einem großen Teil auf Übersichtsarbeiten wie beispielsweise diejenige aus dem Jahr 1998 von Ward, Mattick und Hall, die sich die Mühe machten, weltweit die Erkenntnisse über Substitutionsbehandlungen zu sichten und zu werten [3].

\section{Mortalität - das verdrängte Problem}

Bei unbehandelten Opiatabhängigen wird die Sterberate auf etwa 2,5-3\% pro Jahr geschätzt [4]. Darunter werden alle Todesfälle subsumiert, seien es Intoxikationen, infektionsbedingte Sterbefälle, Unfälle, Suizide und anderweitig bedingte Todesfälle. Vielleicht hat sich diese Zahl mit den neuen HIV-Behandlungen etwas gesenkt, dafür wird nach heutigem Stand des Wissens die Zahl der Hepatitis-C-Toten drastisch zunehmen [5]. Im Vergleich dazu liegt die berichtete Todesrate unter Substitutionsbehandlungen meistens bei rund $1 \%$ pro Jahr [6].

Die Spanne zwischen 1 und 3\% sieht zwar nach einer relativ kleinen Differenz aus, es handelt sich aber um die jährliche Rate. Werden diese Werte auf 10 oder 20 Jahre kumuliert, sieht dies dann sehr dramatisch aus (vgl. Abb.1). 20 Jahre entsprechen beispielsweise dem Altersbereich von 17 bis 37 Jahren. Dies ist bei einem abhängigen Menschen eine normale Zeitspanne in der oftmals mit dem zynischen Begriff „Suchtkarriere“ bezeichneten Lebensphase. Aufgrund dieser Berechnungen ist nach 10 Jahren bereits ein Viertel der Unbehandelten verstorben, nach 20 Jahren sind es ca. $45 \%$. Unter Substitution liegen diese Werte nach 10 Jahren bei maximal einem Zehntel bzw. nach 20 Jahren bei unter $20 \%$.

Am Beispiel der Schweiz sei zur Illustration der problematische Umgang mit Drogentodesstatistiken aufgezeigt. Etwa die Hälfte

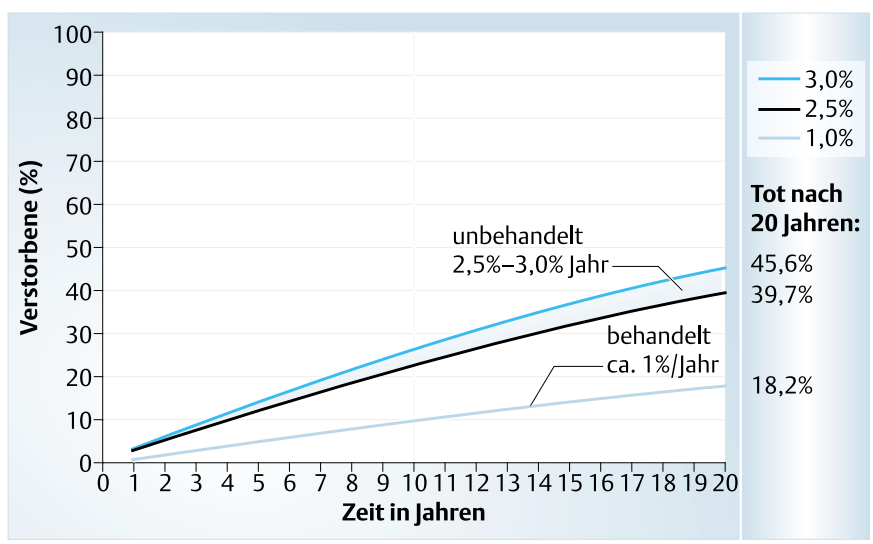

Abb. 1 Verstorbene nach 1 bis 20 Jahren bei Sterberaten von $1 \%$, resp. 2,5-3\% pro Jahr (bei problematischem Konsum von Heroin/ Kokain). 
der 30000 Drogenabhängigen mit problematischem Konsum ist substituiert (im Jahr 2002 waren es insgesamt knapp 18000 Methadon-[7] und rund 1200 Heroinbehandlungen [8] - dies entspricht allerdings nur einer durchschnittlichen Behandlungszahl von $15000-16000$ ). Daneben existieren rund 1000 abstinenzorientierte Behandlungsplätze. Beinahe die Hälfte der Abhängigen befindet sich also nicht in einer Behandlung. In der offiziellen Drogentodesstatistik werden nur vermutete oder sichere Intoxikationen aufgeführt. Im Jahr 2002 waren dies 167 [9]. Nach den Erkenntnissen der Sterberate liegt die Zahl der jährlichen Todesfälle bei Drogenabhängigen aber bei ca. 580; etwa 160 ( $1 \%$ von 16000$)$ bei der Gruppe der Behandelten und ca. 420 (3\% von 14000) bei der Gruppe der Unbehandelten (Estermanns Berechnungen für die 90er-Jahre mit im Durchschnitt 700 Toten lagen sogar noch höher [4]). Dies sind mehr Tote als die gut 500, die der Verkehr in der Schweiz im Jahr 2002 gefordert hat [10].

Wesentlich ist, dass sich die jährliche Mortalitätsrate stark, nämlich auf etwa ein Drittel senken lässt, wenn eine substitutionsgestützte Behandlung mit wenigen Behandlungsauflagen, genügend hoher Dosierung und diversifiziertem professionellen Hilfsangebot stattfindet $[3,6]$.

Zieht man die Parallele zu somatischen Erkrankungen mit einer ähnlich hohen Mortalität, würde man nicht zögern, von einem malignen oder bösartigen Verlauf zu sprechen. Therapeutische Maßnahmen, welche die Sterblichkeit um einen ähnlichen Faktor senken, werden als sehr erfolgreich beurteilt, auch wenn sie nicht zu einer vollständigen Heilung führen.

Die basale Aufgabe der Suchtmedizin muss somit sein, den Abhängigen zu ermöglichen, ihre Krankheit zu überleben.

\section{Abstinenz - das überbewertete Ziel}

Wenden wir uns der Abstinenz zu, stellen wir ernüchtert fest:

Im Langzeitverlauf über 10 bis 20 Jahre gibt es kaum eine Evidenz, dass sich die Abstinenzquote durch irgendwelche therapeutischen Maßnahmen, sei es durch abstinenzorientierte, sei es durch substitutionsgestützte Behandlungen, wesentlich beeinflussen ließe. Es deutet alles darauf hin, dass ein allfälliger Unterschied nach Jahren sukzessive verschwindet. Bei einem Teil der Abhängigen scheint der Konsum selbstlimitierend zu sein.

Als Beispiel für viele sei Patrick O‘Connor von der Yale University School of Medicine zitiert [11]:

„Dennoch gibt es wenig Forschungsresultate über die langfristige Wirksamkeit bezüglich der Opioidabstinenz über die akute Entzugsphase hinaus (einige Tage oder Wochen). Die Effektivität der Entzugsbehandlung ist für die meisten Patienten fraglich, insbesondere für diejenigen, die täglich Heroin konsumieren.“

Dass das Abstinenzparadigma, mindestens in der Light-Version, weiterhin das drogentherapeutische Feld dominiert, zeigt sich zum einen in der Gewichtung der Forschungsbemühungen, zum anderen in den Konzepten oder Leitlinien von Substitutionspro- grammen. Stellvertretend die folgende Aussage zu den Schweizer Heroinverschreibungsprojekten - Ähnliches findet sich auch bei sehr vielen Methadonprogrammen: „Oberstes Ziel in der Suchtbehandlung ist das Erreichen einer selbstständigen und eigenverantwortlichen Lebensführung in Suchtfreiheit“" [12]. Die tägliche Praxis hat dazugeführt, dass in den meisten Programmen mittlerweile die Überlebenssicherung und Steigerung der Lebensqualität im Zentrum stehen.

Doch die bekannten, immer wieder anzutreffenden Stufenmodelle belegen, dass in vielen therapeutischen Einrichtungen im Hintergrund die Abstinenz als letztes Ziel wartet und die Behandlung mitprägt:

1. Eintrittsphase, Integration ins Programm

2. Stabilisierung und Konsolidierung der gesundheitlichen Situation

3. Rehabilitation, Resozialisation

4. Abstinenz und Austritt

Dem ausbleibenden langfristigen Erfolg nach zu schließen, widerspricht eine solche Zielsetzung deutlich dem Realitätsprinzip. Realistischere Erfolgsvorgaben sind notwendig, wenn wir weder unsere selbstgesteckten Ziele dauernd außer Acht lassen noch in „therapeutischem Pessimismus“ [13] versinken wollen.

\section{Kontrollierter Konsum - das unterschätzte Phänomen}

Der kontrollierte Konsum „harter“ Drogen ist ein weiterer Bereich, in dem gut abgestützte Erkenntnisse vorliegen, die aber selbst von vielen Fachleuten kaum zur Kenntnis genommen oder ignoriert werden.

Schippers und Cramer [14], die eine breite Übersichtsarbeit zum Thema kontrollierter Konsum von Drogen verfasst haben, definieren das Phänomen folgendermaßen:

„Kontrollierter Konsum harter Drogen lässt sich demnach als ein Konsum definieren, der nicht in nennenswertem Maß mit persönlichen Zielen kollidiert und durch Selbstkontrollregeln gesteuert wird." Sie beziehen sich auf fünf Reviews von Langzeitstudien mit insgesamt über 30000 Drogenabhängigen und stellen fest, dass nach 10 Jahren ein Viertel zwar immer noch Drogen konsumiert, aber keinen problematischen Umgang damit aufweist. Weber und Schneider [15], die kontrollierte Drogenkonsumenten und Selbstaussteiger verglichen haben, kommen zum Schluss, dass eine solche Entwicklung die Nutzung und Wirksamkeit vorhandener Hilfssysteme, die Antizipation positiver Folgen und die Aktivierung vorhandener Identitätsressourcen voraussetzt. Dass sich der kontrollierte Konsum gezielt fördern lässt, ist somit zu vermuten.

Zu bedenken ist auch, dass selbst während einer stationären Entwöhnungstherapie von illegalen Drogen oder Alkohol (sowohl zu Beginn wie am Ende der Therapiemaßnahme!) für ca. ein Drittel der PatientInnen nicht die totale Abstinenz das Ziel der Wahl ist, sondern ein reduzierter Konsum oder eine nur vorübergehende Abstinenzphase angestrebt wird [16]. Wenn dies bereits bei der Gruppe gilt, die sich in einer langfristigen Entzugsbehandlung 
befindet, so werden alle anderen Konsumenten noch seltener Abstinenz als primäres und realistisches Behandlungsziel sehen!

Wollen wir uns aber an das Prinzip der gemeinschaftlichen Zielsetzung halten - was für eine erfolgreiche Therapie, mindestens im ambulanten Kontext, unabdingbar ist -, so zeigt auch dies, dass wir andere Ziele als die Abstinenz in den Vordergrund stellen müssen. Nämlich Ziele, die aus dem Lebenszusammenhang der PatientInnen heraus Sinn ergeben und für diese selbst erstrebenswert sind.

\section{Prognostische Drittelsregel}

Trotz der spärlichen validen Langzeituntersuchungen wagen wir abzuschätzen, wie die Prognose für Opiatabhängige retrospektiv in den vergangenen Jahrzehnten ausgesehen haben könnte. Betrachtet werden die Mortalität und die Konsummuster 15 bis 20 Jahre nach Konsumbeginn. Zusammenfassend kann von einer „Drittelregel“ ausgegangen werden:

Ein Drittel der Patienten starb, zwei Drittel überlebten. Von den Überlebenden verharrte ein Drittel in dekompensiertem, problematischen Drogenkonsum, ein Drittel wechselte zu einem sporadischen und/oder kontrollierten Konsum und nur ein Drittel erlangte die nachhaltige Abstinenz (vgl. Abb. 2).

\section{Zwischenstand in Kürze}

Bisher wurden drei Dinge aufgezeigt:

\section{Erstens:}

Sucht ist eine chronische Krankheit. Vom Konzept der Heilung auszugehen verkennt die Realität des Verlaufs. In Anbetracht der hohen Mortalität, die als größtmögliche Schädigung betrachtet werden muss, können wir von einem basalen Behandlungsziel sprechen: Drogenabhängigen muss die Möglichkeit geschaffen werden, ihr Überleben zu sichern.

\section{Zweitens:}

Die Abstinenz lässt sich langfristig durch therapeutische Maßnahmen nicht wesentlich beeinflussen. Entsprechend dem Realitätsprinzip kann sie keine sinnvolle und erst noch übergeordnete Zielsetzung sein.

\section{Drittens:}

Der kontrollierte Konsum harter Drogen, auch bei längerer Abhängigkeit, ist vermutlich ebenso häufig wie die Abstinenz nach Abhängigkeit. Beim gemeinsamen Erarbeiten von Zielsetzungen muss dies berücksichtigt werden.

\section{Neue Ausrichtung der Zielhierarchie}

Aus den obigen drei Erkenntniskomplexen sollen im Folgenden die Konsequenzen für die Ausrichtung der therapeutischen $\mathrm{Ar}$ beit aufgezeigt werden.
Die Suchtmedizin hat sich lange Zeit von Zielvorstellungen leiten lassen, wie sie beispielsweise von Körkel und Kruse vor einigen Jahren (1997) in einer Zielpyramide dargestellt wurden (Abb. 3). Aus den neueren Arbeiten dieser Autoren, mit Schwergewicht auf kontrolliertes Trinken bei Alkoholabhängigkeit, geht hervor, dass sie sich von der Abstinenz als oberstem bzw. einzigem Ziel einer Behandlung verabschiedet haben [17-20].

Wir schlagen eine Neuauflage der generellen Zielsetzungen der Suchtbehandlung vor und verzichten auf die Abstinenz als Ziel (Abb. 4).

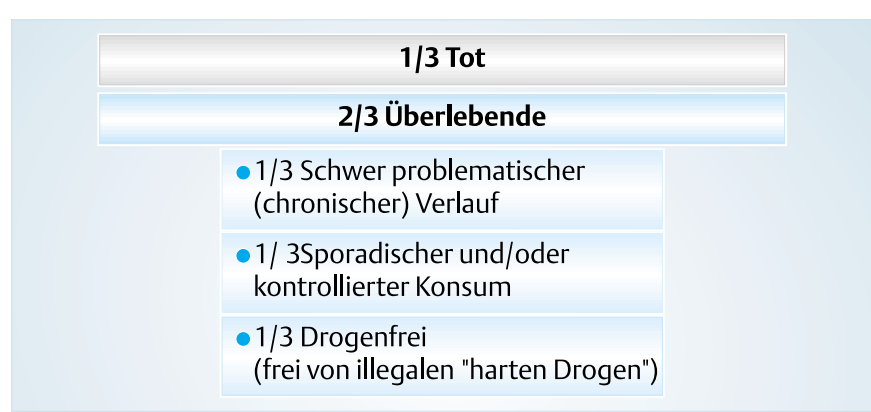

Abb. 2 Drittelregel: geschätzte Langzeitprognose (ca. 20 Jahre) in der Vergangenheit.

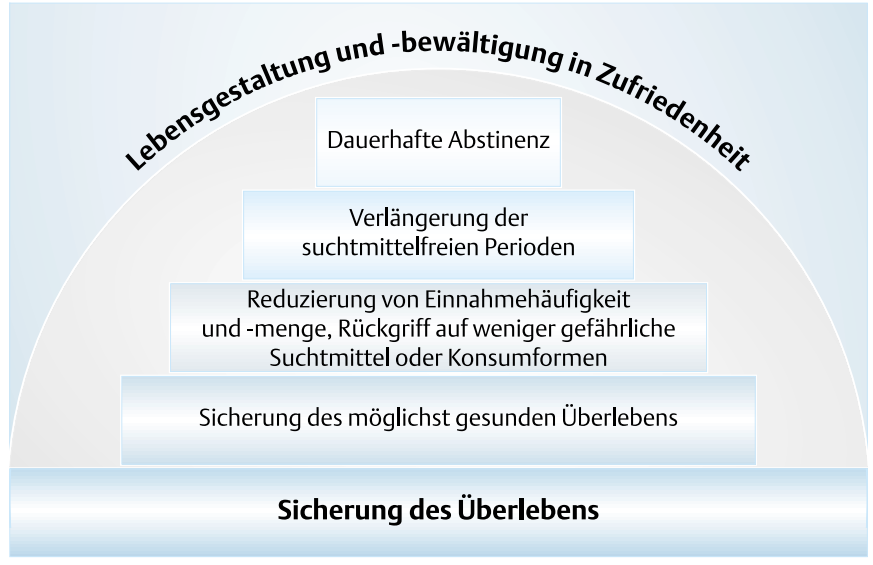

Abb. 3 Klassische Zielpyramide in der Suchttherapie (n. Körkel \& Kruse).

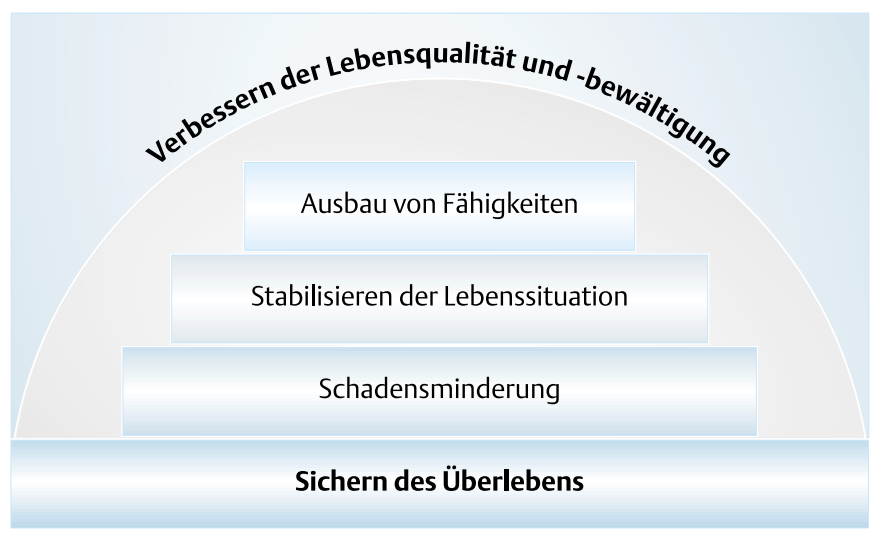

Abb. 4 Zielsetzungen in der Suchttherapie jenseits des Abstinenzparadigmas. 
Die suchtmedizinische Grundversorgung muss den Abhängigen als Basis das Überleben ermöglichen. Alle weiteren Zielsetzungen haben sich an den Ressourcen, am individuellen Hintergrund und den aktuellen Problemstellungen des drogenabhängigen Menschen zu orientieren. Fähigkeiten sollen sich entwickeln können, um Lebensumstände aufzubauen, die sowohl eine größere Frustrationstoleranz ermöglichen als auch verlässlichere Befriedigungsmöglichkeiten, bessere körperliche und psychische Gesundheit und größere soziale Sicherheit bieten.

Schadensminderung, Stabilisieren der Lebenssituation und Ausbau der Fähigkeiten sind Prozesse, die aufeinander aufbauen, sich aber auch parallel entwickeln können. Die Vorstellungen und Wünsche der Abhängigen müssen berücksichtigt und in einem gemeinschaftlichen Entscheidungsprozess integriert werden. Die Zielfindung hat unabhängig davon zu geschehen, was die gesellschaftlichen Erwartungen einfordern, aber abhängig davon, was individuell maximal erreichbar ist. Und hier setzt eine zentrale therapeutische Leistung ein: der undogmatische Diskurs über individuell sinnvolle Zielinhalte und deren Realisierungschance.

Diese Auseinandersetzung muss immer darauf ausgerichtet sein, die Lebensqualität und Lebensbewältigung der Drogenabhängigen zu verbessern, da Entwicklungsschritte nur Bestand haben, wenn daraus eine subjektiv positive Bilanz resultiert.

\section{Therapeutische Methoden}

Die Entwicklung der Unterteilung von Suchttherapien in abstinenzorientierte stationäre und substitutionsgestützte ambulante Behandlungen als Grundsystem ist ein historisch bedingter Irrtum. Denn hier definiert, vor allem im stationären Setting, der Behandlungsrahmen die Hauptzielsetzung, in diesem Fall die Abstinenz.

Ein kategorisches und kategoriales Umdenken ist dringend nötig. Wie bei allen Erkrankungen üblich müssen die optimalen Methoden und Techniken für eine Behandlung in einem geeigneten Rahmen oder Setting zur Verfügung stehen.

Wenn Opiate als zentrale Drogen konsumiert werden, stehen zwei grundlegende Behandlungsmöglichkeiten zur Verfügung: Therapien mit oder ohne Substitution.

Die heute weit gehend praktizierte Dichotomie - substitutionsgestützte ambulante Behandlung versus abstinenzorientierte stationäre Therapie - soll in eine Vierfeldmatrix von Methoden und Settings überführt werden. Einerseits sollen substitutionsgestützte Behandlungen konsequent ambulant wie auch stationär angeboten werden. Andererseits wird eine Methode vorgestellt, in welcher Abhängige mindestens phasenweise nicht unter Drogenwirkung stehen - dies aber nicht mit dem Ziel der Abstinenz. Auch diese Therapieform soll im ambulanten wie stationären Rahmen zur Anwendung gelangen.

\section{Substitutionsgestützte Behandlung}

In der substitutionsgestützten Behandlung wird als Basis eine illegal konsumierte Droge durch eine legal ärztlich verschriebe- ne ersetzt. Die Verwendung der heute gebräuchlichen Substitutionsmittel, wie Methadon, Buprenorphin, Heroin oder anderen Opiaten, erfüllt die Basisforderung der Zielpyramide, indem sich die Mortalitätsrate massiv senken lässt [3,6] und sich Sekundärrisiken, wie Neuansteckungen mit Hepatits B und C und HIV reduzieren $[21,22]$.

\section{Ambulant}

Die substitutionsgestützte ambulante Behandlung als weit gehend bekannte Methode ist sicher die zentrale Säule der Überlebenshilfe bei Opiatabhängigen. Die wichtigsten Modifikationen, die vielerorts vorgenommen werden müssten, sind: definitives Verabschieden von der Abstinenz als Ziel, Senken der Eintrittsschwelle, höhere Dosen, flexiblerer und eigenverantwortlicherer Umgang mit der Dosierung, weniger obligatorische Auflagen an die Betreuung, Ausbau des fakultativen Angebots an medizinischer, d.h. psychiatrischer und vor allem auch somatischer Betreuung. Der soziale Aspekt ist meistens genügend berücksichtigt.

\section{Stationär}

Für eine Gruppe Abhängiger in desolatem psychischen, körperlichen und/oder sozialen Zustand, für die keine andere Behandlungsmethode geeignet ist, sollen stationäre substitutionsgestützte Behandlungen geschaffen werden. Der beschrittene Weg soll dahin führen, dass die Behandlung in eine stabile ambulante substitutionsgestützte Therapie überführt werden kann. Beispielsweise könnte die Grundlage dafür erarbeitet werden, dass eine Patientin nach Austritt aus der stationären Einrichtung einer regelmäßigen Tätigkeit nachgehen oder ihre Kinder adäquat versorgen kann. Neben verschiedenen Therapieformen würden wie auch Aus- und Weiterbildungselemente helfen, die soziale Kompetenz zu erhöhen.

Daneben könnten somatische Zielsetzungen in den Fokus genommen werden, zum Beispiel die Durchführung oder das Einleiten einer Hepatitis-C-Therapie oder das Einstellen einer HIVBehandlung, sofern dies ambulant nicht gelingt. Hierfür würden sich therapeutische Gemeinschaften mit Einbezug kompetenter medizinischer Versorgung hervorragend eignen.

Vom Damoklesschwert der Abstinenz befreit, dürften unter Substitution im stationären Rahmen viele Reifungsprozesse eher gelingen als in den herkömmlichen abstinenzorientierten Settings. Die Dauer der Behandlung müsste den Zielsetzungen entsprechend individuell geplant werden können.

\section{Abstinenzgestützte Behandlung}

Neben der substitutionsgestützten Behandlung wird eine zweite Behandlungsmethode vorgestellt. Dazu soll ein neuer Terminus eingeführt werden, der aus anderem Munde stammt und für die hier wiedergegebene Haltung sehr treffend erscheint. Thomas Meyer, Chefarzt der Forelklinik in der Schweiz (einer Klinik, in der Alkoholabhängige behandelt werden), äußerte im Frühjahr 2002 in einem Referat über Therapieziele im Suchtbereich Folgendes: „Abstinenz ist kein Behandlungsziel, sondern, wenn schon, eine Behandlungsmethode; Stichwort: abstinenzgestützte Therapie“" (mündliche Mitteilung vom 13.3.2002). 
Abstinenzorientiert oder sinnverwandte Begriffe wie Abstinenzbehandlung, denen die Abstinenz als Hauptziel gemeinsam ist, sollen aufgegeben werden. Analog zur Methode der substitutionsgestützten Behandlung soll diejenige der abstinenzgestützten Behandlung konzeptualisiert werden.

Bei der abstinenzgestützten Behandlung werden Phasen angestrebt, in denen die Abhängigen nicht unter Drogenwirkung stehen. Die Länge dieser Phasen soll individuell festgelegt und je nach Lebensumständen angepasst werden. In diesen Phasen kann - ebenso wie unter stabiler Substitution - einerseits die Auseinandersetzung mit eigenen Konsummustern und Konsumformen gefördert und andererseits können andere therapeutische Ziele, die zur Stabilisierung der Lebenssituation und Verbesserung der Lebensqualität beitragen, verfolgt werden. Ein positiver Effekt, der erreicht werden kann, ist ein stabiler kontrollierter Konsum, „der nicht in nennenswertem Maße mit persönlichen Zielen kollidiert und durch Selbstkontrollregeln gesteuert wird und im Hinblick auf Menge und Häufigkeit limitiert und hinsichtlich der Konsequenzen unproblematisch“ ist [14]. Ebenso können weniger riskante Konsumformen gesucht werden. Angestrebt wird das individuell höchste Kontrollniveau, welches aktuell erreicht werden kann und eine größtmögliche Stabilisierung der Lebenssituation ermöglicht. Die maximale Kontrolle des Konsums ist die anhaltende Abstinenz, die als längst mögliche Phase ohne Drogenwirkung betrachtet werden kann.

Der Drogenkonsum, auch nach längerer Abstinenz, sollte weder als Rückfall noch als Misserfolg bewertet werden, sondern als Beikonsum zur Abstinenz, analog zum Beikonsum zur Substitution. Gerade bei längerer drogenfreier Zeit ist es äußerst wichtig, Vorkehrungen zu treffen, um sicherzustellen, dass sich bei erneutem Konsum die Kontrolle aufrecht erhalten lässt und ein Eintauchen in die Spirale des unkontrollierten Konsums verhindert werden kann. Dies ist unter anderem wegen der physiologischen Toleranzbildung auf Drogen notwendig.

\section{Ambulant}

Die ambulante abstinenzgestützte Behandlung kann nach einer langsamen Reduktion eines Substitutionsmittels, nach einem rascheren ambulanten oder stationären „körperlichen“ Entzug (mit oder ohne medikamentöse Unterstützung bzw. Substitutionsmittel), nach einer stationären abstinenzgestützten Behandlung oder ohne Vorbehandlung direkt zur Anwendung gelangen.

Um die Kontrolle des Konsums zu vergrößern, sind oft kognitivverhaltenstherapeutische Elemente hilfreich, wie sie auch in Programmen zum kontrollierten Alkoholkonsum angewendet werden.

Günstig kann die ambulant angewandte Methode speziell bei Berufstätigen oder sozial Gebundenen sein, z.B. bei Patienten mit Kindern. Geeignet ist sie aber auch für Abhängige, die den Konsum nicht so radikal verändern können oder wollen, wie es in einem stationären Setting notwendig wäre (s. unten). Im ambulanten Bereich kann beispielsweise eine Reduktion von sechs intravenösen Applikationen auf eine pro Tag bereits als minimale abstinenzgestützte Intervention bezeichnet werden, die sehr erfolgreich hinsichtlich Schadensminderung ist.

Eigene Erfahrungen liegen im Einzelsetting sowohl mit Opiat- als auch mit Kokainabhängigen und solchen mit polytoxikomanem Konsummuster vor. Da bei Kokainabhängigen die Substitution keine legale Option darstellt, kommt die abstinenzgestützte Behandlung hier häufiger zum Zuge. Es zeigt sich, dass die unterschiedlichsten kontrollierten oder zumindest kontrollierteren Konsummuster Bestand haben können.

\section{Stationär}

Die stationäre abstinenzgestützte Behandlung ist vermutlich für alle als Therapieversuch geeignet, die sich auf diesen Rahmen einlassen wollen. Wir müssen uns dabei bewusst sein, dass wir den Patienten nur Empfehlungen über sinnvolle Behandlungsmethoden und -settings abgeben können, sie aber ihre Wege und scheinbaren Umwege selbst bestimmen. Nur dann kann auch das Prinzip der gemeinschaftlichen Zielsetzung wirklich Anwendung finden.

$\mathrm{Zu}$ Beginn einer solchen Behandlung muss gegenwärtig eine starke Konsumreduktion vorausgesetzt oder sogleich angestrebt werden, da aufgrund der Praktikabilität und aus rechtlichen Gründen der Konsum illegaler Drogen in der jeweiligen Institution kaum tolerierbar ist.

Mit den Abhängigen können Strategien erarbeitet werden, die darauf abzielen, dass die Chance steigt, nicht mehr zu konsumieren (maximale Kontrolle) oder kontrolliert zu konsumieren. Wird der Konsum nicht als Rückfall, sondern als optimal zu kontrollierender Beikonsum gewertet, macht die bei abstinenzorientierten Therapien oftmals lange Abschirmung von der Außenwelt kaum Sinn. Die frühzeitige Konfrontation mit Konsum oder Konsummöglichkeiten soll fester Bestandteil der Behandlung sein. Erfolgreich zu sein, kann dann bedeuten, den Konsum im Ausgang zu reduzieren, beispielsweise auf einmal pro Woche oder auf das Wochenende und diese Frequenz nach Austritt nicht wieder auszudehnen.

Ein Behandlungsrahmen, der gegen außen zu stark schützt und in welchem diese Exposition nicht möglich ist, kann eine Atmosphäre schaffen, in der sich eine vermeintliche Sicherheit entwickelt, abstinent zu bleiben. Gruppenprozesse fördern allzu gerne das Gefühl „wir haben es geschafft und sind keine Junkies mehr“. Ein allfälliger späterer Konsum kommt dann häufig unvorbereitet, d.h. ohne internalisierte Strategie zur Selbstlimitierung, und hat oftmals ein lebensgefährliches Ausmaß [23].

Das Argument, die Thematisierung eines möglichen Konsums auch bei länger dauernder Abstinenz fördere den Konsum, ist vermutlich ebenso wenig stichhaltig, wie die Angst berechtigt ist, das Ansprechen auf Suizidalität erhöhe die Suizidgefahr.

Der Austritt aus der stationären abstinenzgestützten Behandlung mit Drogenkonsum ist somit nicht mehr ein grundsätzlicher Misserfolg und derjenige ohne Drogenkonsum ein Erfolg. Beides kann mit dem Erreichen von vorher festgelegten sinnvollen Zielen verknüpft sein. 
Aus all diesen Überlegungen resultiert eine Ergänzung der Pyramide mit Integration der beiden therapeutischen Basismethoden und des kontrollierten Konsums (Abb.5).

\section{Abhängigkeit kennt keine lineare Entwicklung}

Da der Verlauf des Drogenkonsums beinahe nie durch eine lineare Entwicklung gekennzeichnet ist, muss gewährleistet sein, dass die einzelnen Methoden und Settings - je nach Lebensumständen und Problemstellungen - frei ineinander überführt werden können. Sinnvoll erscheint, dass ambulante und stationäre Institutionen je beide Methoden - substitutions- und abstinenzgestützt - anbieten, damit nicht bei jeder Veränderung ein Institutionswechsel nötig wird und somit die Konstanz der Behandlung gesichert werden kann. Möglicherweise könnte es sogar optimal sein, wenn alle vier Systeme unter einem Dach offeriert würden. Die Konfrontation des einzelnen Abhängigen mit

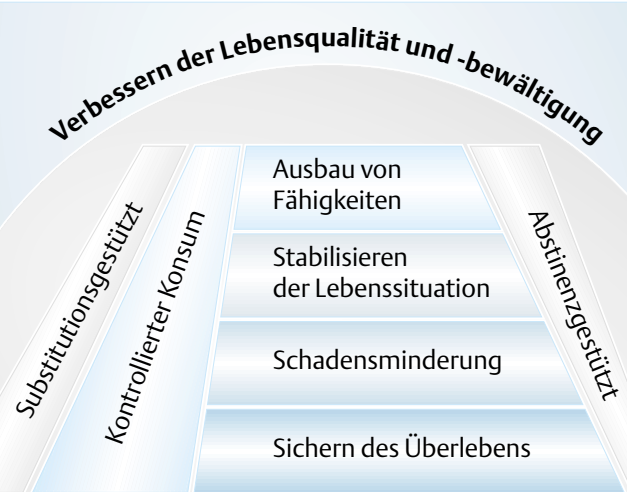

Abb. 5 Zielsetzungen in der Suchttherapie in Kombination mit Behandlungsmethoden.

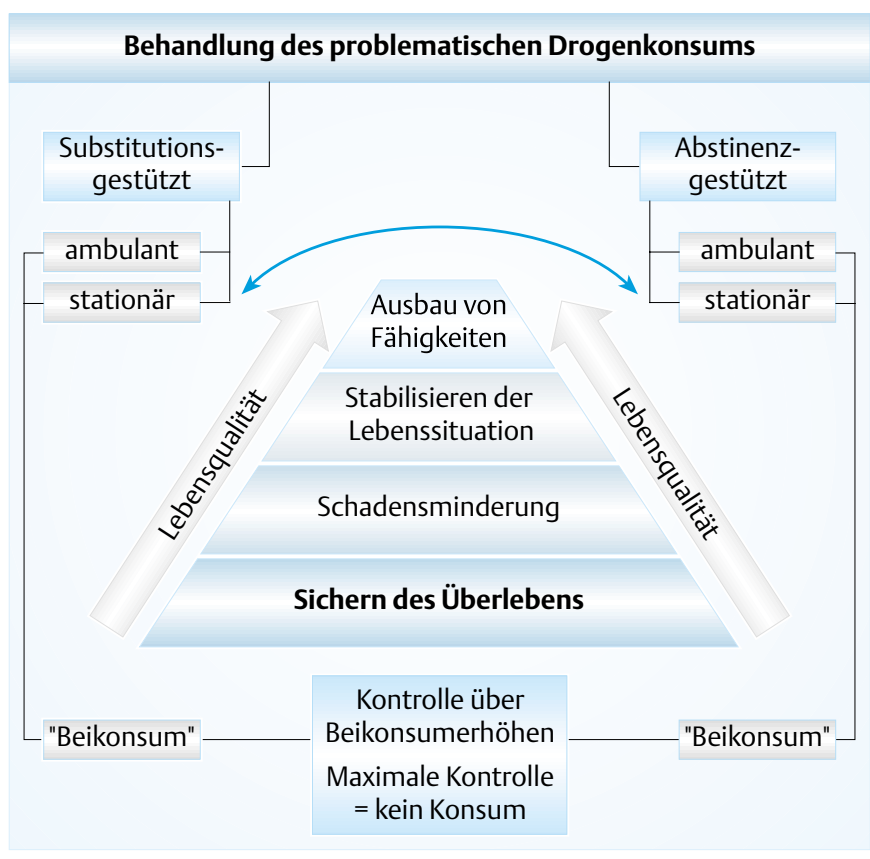

Abb. 6 Zielsetzungen, Behandlungsmethoden und Settings in einer Suchttherapie jenseits des Abstinenzparadigmas. verschiedenen Methoden und Settings könnte durchaus konzeptionell eingesetzt werden, um sich von Standardlösungen zu distanzieren und dem einzelnen Menschen zu ermöglichen, einen individuellen, maßgeschneiderten Weg zu beschreiten. Eine zusammenfassende schematische Darstellung der Zielsetzungen, Behandlungsmethoden und Settings findet sich in Abb. 6. Zahlenmäßig wird das Schwergewicht auch zukünftig auf dem ambulanten Bereich liegen, in beiden Settings vermutlich stärker auf der substitutionsgestützten Methode.

\section{Fazit}

Erst wenn wir lernen, uns in der Suchtmedizin vom dogmatischen Ziel der Abstinenz vollständig zu verabschieden, ist es möglich, individuelle Ziele gemeinsam mit den Abhängigen auf ihre Machbarkeit hin zu überprüfen, zu diskutieren, Modifikationen vorzuschlagen, neue Ideen einzubringen und Wege zusammen zu beschreiten, deren Ende wir nicht kennen. Auf diese Weise können die Abstinenz von Beikonsum oder die Abstinenz generell zu einem sinnvollen Nebenprodukt werden. Als Ziel zieht das „Irrlicht Abstinenz“ aber die Aufmerksamkeit auf sich und lässt einen allzu oft vom Weg abkommen, was im Abgrund enden kann - für die Abhängigen zuweilen tödlich.

Zum Schluss kann eine von uns aufgestellte Behauptung relativiert werden: Selbstverständlich lässt sich die Zahl der Abstinenten beeinflussen, und zwar indem die Behandelnden sich darauf konzentrieren, die Überlebenschance der Abhängigen zu steigern. Denn: Tote konsumieren zwar keine Drogen, aber nur Lebende können abstinent werden.

\section{Literatur}

${ }^{1}$ McLellan AT. Have we evaluated addiction treatment correctly? Implications from a chronic care perspective. Addiction 2002; 97 (3): 249-252

2 McLellan AT, Lewis DC, O'Brien CP et al. Drug dependence, a chronic medical illness: implicatins for treatment, insurance, and outcomes evaluation. JAMA 2000; 284 (13): 1689-1695

${ }^{3}$ Ward J, Mattick RP, Hall W. Methadone maintenance treatment and other opioid replacement therapies. Amsterdam: Harwood Academic Publishers, 1998

${ }^{4}$ Estermann J. Sozialepidemiologie des Drogenkonsums. Berlin: Verlag für Wissen und Bildung, 1996

${ }^{5}$ Grob PJ. Schweiz. Expertengruppe für virale Hepatitis (SEVHEP). Infektionen mit dem Hepatitis C Virus Epidemiologie, Prävention und Behandlung. BAG-Bulletin 1998; 49: 2-12

${ }^{6}$ Desmond DP, Maddux JF. Deaths among heroin users in and out of methadone treatment. J Maintenance in the Addictions 2000; 1: 45-61

${ }^{7}$ BAG-2003 Bundesamt für Gesundheitswesen. Nationale Substitutionsstatistik (Methadon). http://www.nasuko.ch/nms/db/index.cfm

${ }^{8}$ BAG-2003 Bundesamt für Gesundheitswesen. Heroingestützte Behandlung. http://www.suchtundaids.bag.admin.ch/themen/sucht/dro gen/unterebenen/00615/index.html

9 BAP-2002 Bundesamt für Polizeiwesen. Drogentote 1992-2001. http://www.bap.admin.ch/d/aktuell/stat/drogen/tote2002.htm

${ }^{10}$ BFS-2002 Bundesamt für Statistik. Unfälle im Straßenverkehr. www.statistik.admin.ch/stat_ch/ber11/dufr11.htm

${ }^{11}$ O'Connor PG. Treating opioid dependance - new data and new opportunities. New England Journal of Medicine 2000; 18: 1332-1333

12 BAG Bundesamt für Gesundheitswesen. Bericht zu den Schweizer Heroinabgabeprojekten, 1997

${ }^{13}$ Hall M. Perfection in the therapeutic appraisal of methadone maintenance. Addiction 1993; 88: 1181- 1182 
${ }^{14}$ Schippers GM, Cramer E. Kontrollierter Gebrauch von Heroin und Kokain. Suchttherapie 2002; 3: 71-80

${ }^{15}$ Weber G, Schneider W. Herauswachsen aus der Sucht illegaler Drogen, Selbstheilung, kontrollierter Gebrauch und therapiegestützter Ausstieg. Münster: Institut für Soziologie/Sozialpädagogik - Westfälische Wilhelms-Universität, 1992

${ }^{16}$ Körkel J, Schindler C. Ziele und Zielvereinbarungen in der Suchtarbeit. In: Fachverband Sucht (Hrsg). Suchtbehandlung. Entscheidungen und Notwendigkeiten. Geesthaacht: Neuland, 1999: 174-196

${ }^{17}$ Körkel J. Vom Sollen zum Wollen: Zielfestlegungen und Zielvereinbarungen in der Suchthilfe. Abhängigkeiten 2000; 6: 5-25

${ }^{18}$ Körkel J. Kontrolliertes Trinken: Eine Übersicht. Suchttherapie 2002; 3: $87-96$

${ }^{19}$ Kruse G, Körkel J, Schmalz U. Alkoholismus erkennen und behandeln. Mit literarischen Beispielen. Bonn: Psychiatrie-Verlag, 2000
${ }^{20}$ Körkel J, Kruse G. Mit dem Rückfall leben. Abstinenz als Allheilmittel? 4., überarbeitete Aufl. Bonn: Psychiatrie-Verlag, 2000

${ }^{21}$ Steffen T. Die infektiologische Situation bei intravenös Drogenabhängigen in den Versuchen für eine ärztliche Verschreibung von Betäubungsmitteln (PROVE) unter besonderer Berücksichtigung von Public Health-Aspekten. Zürich: Institut für Suchtforschung, 1998

22 Moss AR, Vranizan K, Gorter R et al. HIV seroconversion in intravenous drug users in San Francisco 1995-1990. AIDS 1994; 8: 223-231

${ }^{23}$ Zinkler M, Valdes J, von Cranach $\mathrm{M}$ et al. Katamnestische Untersuchung niedrigschwellig entgifteter Opiatabhängiger. Sucht 1998; 44 (1): $25-33$ 